\title{
NUMERICAL APPROXIMATION FOR INTEGRAL EQUATIONS
}

\section{ELIAS DEEBA and SHISHEN XIE}

\author{
Received 15 July 2003
}

\begin{abstract}
A numerical algorithm, based on a decomposition technique, is presented for solving a class of nonlinear integral equations. The scheme is shown to be highly accurate, and only few terms are required to obtain accurate computable solutions.
\end{abstract}

2000 Mathematics Subject Classification: 45G10, 41A10, 65R20.

1. Introduction. Adomian polynomial algorithm has been extensively used to solve linear and nonlinear problems arising in many interesting applications (see, e.g., [1, $2,4,5]$ ). The algorithm (a decomposition method) assumes a series solution for the unknown quantity. It has been shown [3] that the series converges fast, and with only few terms, this series approximates the exact solution with a fairly reasonable error. In this note, we will adapt the algorithm and a modification version of the algorithm due to Wazwaz [7] to the solution of the nonlinear Volterra-Fredholm integral equations arising in the modeling of many applications [8]:

$$
y(x)=f(x)+\lambda_{1} \int_{a}^{x} K_{1}(x, t) g_{1}(y(t)) d t+\lambda_{2} \int_{a}^{b} K_{2}(x, t) g_{2}(y(t)) d t,
$$

and analyze the solution. In (1.1), where $K_{1}(x, t)$ and $K_{2}(x, t)$ are referred to as the kernel, $g_{1}$ and $g_{2}$ are nonlinear functions of $y$, and $f(x)$ a given function, $g, K$, and $f$ are known functions, and $\lambda_{1}$ and $\lambda_{2}$ are parameters.

The balance of this note is as follows. In Section 2, we describe the general algorithm as it applies to the solution of integral equations of the form (1.1). In Section 3, we adapt the algorithm to some problems.

2. Analysis. In this section, we first describe the algorithm of the decomposition method as it applies to a general nonlinear equation of the form

$$
y=f+N(y)
$$

or

$$
y-N(y)=f,
$$

where $N$ is a nonlinear operator on a Hilbert space $H$ and $f$ is a known element of $H$. We assume that for a given $f$, a unique solution $u$ of (2.2) exists. 
The standard decomposition method assumes a series solution for $u$ given by

$$
y=\sum_{n=0}^{\infty} y_{n}=y_{0}+y_{1}+y_{2}+\cdots
$$

and the nonlinear operator $N$ can be decomposed into

$$
N(y)=\sum_{n=0}^{\infty} A_{n}
$$

where the $A_{n}$ 's are Adomian's polynomials of $y_{0}, \ldots, y_{n}$ given by

$$
A_{n}=\frac{1}{n !} \frac{d^{n}}{d \lambda^{n}}\left[N\left(\sum_{i=0}^{\infty} \lambda^{i} y_{i}\right)\right]_{\lambda=0}, \quad n=0,1, \ldots
$$

Substituting (2.3) and (2.4) into the functional equation (2.2) yields

$$
\sum_{n=0}^{\infty} y_{n}-\sum_{n=0}^{\infty} A_{n}=f
$$

If the series in (2.6) is convergent, then (2.6) holds upon setting

$$
\begin{gathered}
y_{0}=f, \\
y_{1}=A_{0}\left(y_{0}\right), \\
y_{2}=A_{1}\left(y_{0}, y_{1}\right), \\
\vdots \\
y_{n}=A_{n-1}\left(y_{0}, y_{1}, \ldots, y_{n-1}\right),
\end{gathered}
$$

Thus, one can recursively determine every term of the series $\sum_{n=0}^{\infty} y_{n}$. The convergence of this series has been established (see [2]). The two hypotheses necessary for proving convergence of the decomposition method as given in [2] are as follows.

Condition 2.1. The nonlinear functional equation (2.2) has a series solution $\sum_{n=0}^{\infty} y_{n}$ such that $\sum_{n=0}^{\infty}(1+\epsilon)^{n}\left|y_{n}\right|<\infty$, where $\epsilon>0$ may be very small.

CondiTION 2.2. The nonlinear operator $N(y)$ can be developed in series $N(y)=$ $\sum_{n=0}^{\infty} \alpha_{n} y^{n}$.

These hypotheses, for proving convergence, are generally satisfied in physical problems.

The modified Adomian method [7] may be roughly described as a reassignment of the initial approximants $y_{0}$ and $y_{1}$. In particular, if $f$ is split into two functions, say, 
$f=f_{1}+f_{2}$, then we may rewrite (2.7) as

$$
\begin{gathered}
y_{0}=f_{1}, \\
y_{1}=f_{2}+A_{0}\left(y_{0}\right), \\
y_{2}=A_{1}\left(y_{0}, y_{1}\right),
\end{gathered}
$$

The choice of how to assign $y_{0}$ and $y_{1}$ is experimental, yet it leads to less computation and does accelerate the convergence.

We now describe the application of the decomposition method to an integral equation of the form (1.1). For the sake of simplicity, we will present the method for integral equations of the form

$$
y(x)=f(x)+\int_{a}^{b} K(x, t) g(y(t)) d t .
$$

The adaption for the method to (1.1) is immediate.

Assuming that $g(y)$ is analytic (and thus satisfying Condition 2.2), we can write

$$
g(u)=\sum_{k=0}^{\infty} A_{k}\left(y_{0}, y_{1}, \ldots, y_{k}\right),
$$

where $A_{k}$ are the specially generated Adomian polynomials which can be constructed by the following procedures.

Assume that the Taylor expansion of $g(y)$ around $y_{0}$ exists and is determined by

$$
g(y)=g\left(y_{0}\right)+g^{(1)}\left(y_{0}\right)\left(y-y_{0}\right)+\frac{1}{2 !} g^{(2)}\left(y_{0}\right)\left(y-y_{0}\right)^{2}+\cdots .
$$

Substituting the difference $y-y_{0}$ from (2.3) into (2.11), we get

$$
\begin{aligned}
g(y)= & g\left(y_{0}\right)+g^{(1)}\left(y_{0}\right)\left(y_{1}+y_{2}+\cdots\right) \\
& +\frac{1}{2 !} g^{(2)}\left(y_{0}\right)\left(y_{1}+y_{2}+\cdots\right)^{2}+\cdots .
\end{aligned}
$$

After expanding, this results in

$$
\begin{aligned}
g(y)= & g\left(y_{0}\right)+g^{(1)}\left(y_{0}\right)\left(y_{1}+y_{2}+\cdots\right) \\
& +\frac{1}{2 !} g^{(2)}\left(y_{0}\right)\left(y_{1}^{2}+2 y_{1} y_{2}+2 y_{1} y_{3}+y_{2}^{2}+2 y_{2} y_{3}+y_{3}^{2}+\cdots\right) \\
& +\frac{1}{3 !} g^{(3)}\left(y_{0}\right)\left(y_{1}^{3}+3 y_{1}^{2} y_{2}+3 y_{1}^{2} y_{3}+3 y_{1} y_{2}^{2}+3 y_{1} y_{3}^{2}+\cdots\right) \\
& +\cdots .
\end{aligned}
$$

Adomian polynomials are obtained by reordering and rearranging the terms given in (2.13). Indeed, to determine the Adomian polynomial, one needs to determine the order of each term in (2.13) which actually depends on both the subscripts and the exponents of the $y_{n}$ 's. To be more specific, we define the order of the component $y_{k}^{m}$ to be $m k$, 
and $y_{k}^{m} y_{j}^{n}$ to be $m k+n j$. Then the Adomian's polynomial $A_{0}$ depends upon $y_{0}$ with order $0, A_{1}$ depends upon $y_{0}$ and $y_{1}$ with order 1 , and so forth. Therefore, rearranging the terms in the expansion equation (2.13) according to the order, we will have $A_{n}$ as follows:

$$
\begin{gathered}
A_{0}=g\left(y_{0}\right), \\
A_{1}=y_{1} g^{(1)}\left(y_{0}\right), \\
A_{2}=y_{2} g^{(1)}\left(y_{0}\right)+\frac{y_{1}^{2}}{2 !} g^{(2)}\left(y_{0}\right), \\
A_{3}=y_{3} g^{(1)}\left(y_{0}\right)+y_{1} y_{2} g^{(2)}\left(y_{0}\right)+\frac{y_{1}^{3}}{3 !} g^{(3)}\left(y_{0}\right),
\end{gathered}
$$

Once the $A_{n}$ are determined by (2.14), one can recurrently determine the terms $y_{n}$ of the series from (2.7), and hence the solution $y$. It is easy to verify that when $N(y)$ is $g(y)$, formula (2.5) yields the same result as in (2.14).

For a detailed description of the decomposition method, we refer the reader to [1, 2, 6].

Substituting (2.3) and (2.10) into (1.1), we have

$$
y_{0}+y_{1}+y_{2}+\cdots=f(x)+\int_{a}^{b} K(x, t)\left(A_{0}+A_{1}+A_{2}+\cdots\right) d t
$$

If the series is convergent, then we can determine each term of the series $\sum_{n=0}^{\infty} y_{n}$ recursively:

$$
\begin{gathered}
y_{0}=f(x), \\
y_{1}=\int_{a}^{b} K(x, t) A_{0}\left(y_{0}\right) d t \\
y_{2}=\int_{a}^{b} K(x, t) A_{1}\left(y_{0}, y_{1}\right) d t \\
\vdots \\
y_{n}=\int_{a}^{b} K(x, t) A_{n-1}\left(y_{0}, y_{1}, \ldots, y_{n-1}\right) d t
\end{gathered}
$$

The algorithm in (2.16) determines the $y_{i}$ 's and hence the solution $y$ can be determined by (2.3). The decomposition method can be applied to solve problems in higher dimensions (see [2]). We will also apply the modified decomposition by writing $f=f_{1}+f_{2}$ with appropriate choice for $y_{0}$ and $y_{1}$. 
TABLE 3.1

\begin{tabular}{c|cccccc}
\hline$x$ & 0 & 0.2 & 0.4 & 0.6 & 0.8 & 1.0 \\
\hline Error & 0 & 0 & $10^{-10}$ & $5 \times 10^{-9}$ & $8.85 \times 10^{-8}$ & $1.41 \times 10^{-7}$ \\
\hline
\end{tabular}

3. The solution of integral equations. In this section, we apply the algorithms described in Section 2 to some problems of integral equations. Most of the problems discussed were solved using Taylor-type method in [8]. The decomposition method is an alternative method for solving these equations. Whenever appropriate, we will note the comparison.

Problem 3.1. We apply the standard decomposition method

$$
y(x)=-\frac{15}{56} x^{8}+\frac{13}{14} x^{7}-\frac{11}{10} x^{6}+\frac{9}{20} x^{5}+x^{2}-x+\int_{0}^{x}(x+t) y^{3}(t) d t
$$

Let $y_{0}(x)=-(15 / 56) x^{8}+(13 / 14) x^{7}-(11 / 10) x^{6}+(9 / 20) x^{5}+x^{2}-x$, then

$$
\begin{gathered}
y_{1}(x)=\int_{0}^{x}(x+t) y_{0}^{3}(t) d t \\
y_{2}(x)=\int_{0}^{x}(x+t) 3 y_{0}^{2}(t) y_{1}(t) d t \\
y_{3}(x)=\int_{0}^{x}(x+t)\left[3 y_{0}^{2}(t) y_{2}(t)+3 y_{0}(t) y_{1}(t)\right] d t \\
y_{4}(x)=\int_{0}^{x}(x+t)\left[3 y_{0}^{2}(t) y_{3}(t)+6 y_{0}(t) y_{1}(t) y_{2}(t)+y_{1}^{3}(t)\right] d t
\end{gathered}
$$

$y(x)$ is approximated by using only five terms of decomposition polynomials:

$$
y(x) \approx y_{0}(x)+y_{1}(x)+y_{2}(x)+y_{3}(x)+y_{4}(x)
$$

The exact solution of the integral equation is $y(x)=x^{2}-x$. Comparing the approximate solution from the decomposition method with the exact solution of the integral equation at $x=0,0.2,0.4,0.6,0.8$, and 1.0 , we find the errors displayed in Table 3.1.

Figure 3.1 shows the approximate and analytic solutions of the equation. The solid curve represents the approximate solution, while the rhombuses represent the analytic solution. It is obvious from the figure that the approximation is very good, although we used only four terms of decomposition polynomials.

Problem 3.2. We apply the standard decomposition method

$$
y(x)=e^{x}-\frac{1}{3} e^{3 x}+\frac{1}{3}+\int_{0}^{x}[y(t)]^{3} d t
$$




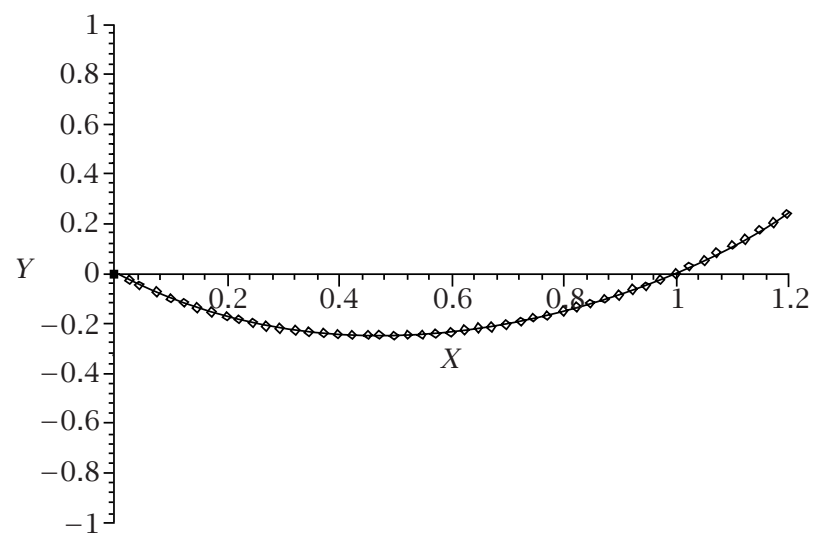

FIGURE 3.1. Decomposition method versus analytic solution for $y(x)=$ $-(15 / 56) x^{8}+(13 / 14) x^{7}-(11 / 10) x^{6}+(9 / 20) x^{5}+x^{2}-x+\int_{0}^{x}(x+t) y^{3}(t) d t$.

Let $y_{0}(x)=e^{x}$, then

$$
\begin{aligned}
y_{1}(x)= & -\frac{1}{3} e^{3 x}+\frac{1}{3}+\int_{0}^{x} y_{0}^{3}(t) d t \\
y_{2}(x)= & \int_{0}^{x} 3 y_{0}^{2}(t) y_{1}(t) d t \\
y_{3}(x)= & \int_{0}^{x}\left[3 y_{0}^{2}(t) y_{2}(t)+3 y_{0}(t) y_{1}^{2}(t)\right] d t \\
y_{4}(t)= & \int_{0}^{x}\left[3 y_{0}^{2}(t) y_{3}(t)+6 y_{0}(t) y_{1}(t) y_{2}(t)+y_{1}^{3}(t)\right] d t \\
y_{5}(x)= & \int_{0}^{x}\left[y_{0}^{2}(t) y_{4}(t)+3 y_{0}(t) y_{2}^{2}(t)+3 y_{1}^{2}(t) y_{2}(t)+6 y_{0}(t) y_{1}(t) y_{3}(t)\right] d t, \\
y_{6}(x)= & \int_{0}^{x}\left[3 y_{1}^{2}(t) y_{3}(t)+3 y_{0}^{2}(t) y_{5}(t)+3 y_{1}(t) y_{2}^{2}(t)+6 y_{0}(t) y_{2}(t) y_{3}(t)\right. \\
& \left.\quad+6 y_{0}(t) y_{1}(t) y_{4}(t)\right] d t \\
y_{7}(x)= & \int_{0}^{x}\left[6 y_{0}(t) y_{2}(t) y_{4}(t)+6 y_{1}(t) y_{2}(t) y_{3}(t)+6 y_{0}(t) y_{1}(t) y_{5}(t)\right. \\
& \left.\quad+3 y_{0}^{2}(t) y_{6}(t)+3 y_{0}(t) y_{3}^{2}(t)+3 y_{1}^{2}(t) y_{4}(t)+y_{2}^{3}(t)\right] d t, \\
y_{8}(x)= & \int_{0}^{x}\left[6 y_{0}(t) y_{1}(t) y_{6}(t)+3 y_{0}^{2}(t) y_{7}(t)+6 y_{0}(t) y_{3}(t) y_{4}(t)\right. \\
& \left.+3 y_{1}^{2}(t) y_{5}(t)+3 y_{1}(t) y_{3}^{2}(t)+3 y_{2}^{2}(t) y_{3}(t)+6 y_{0}(t) y_{2}(t) y_{5}(t)\right] d t
\end{aligned}
$$

$y(x)$ is approximated by using nine terms of decomposition polynomials:

$$
\begin{aligned}
y(x) \approx & y_{0}(t)+y_{1}(t)+y_{2}(t)+y_{3}(t)+y_{4}(t) \\
& +y_{5}(t)+y_{6}(t)+y_{7}(t)+y_{8}(t) .
\end{aligned}
$$


TABLE 3.2

\begin{tabular}{c|cccccc}
\hline$x$ & 0 & 0.2 & 0.4 & 0.6 & 0.8 & 1.0 \\
\hline Error & 0 & $7.1 \times 10^{-11}$ & $3.3 \times 10^{-10}$ & $2.5 \times 10^{-9}$ & $2.3 \times 10^{-8}$ & $3.8 \times 10^{-7}$ \\
\hline
\end{tabular}

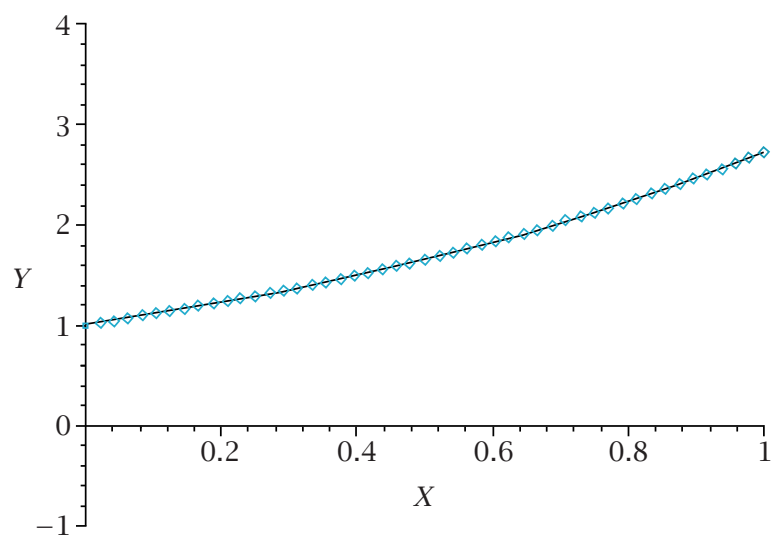

FIGURE 3.2. Decomposition method versus analytic solution for $y(x)=e^{x}-$ $(1 / 3) e^{3 x}+1 / 3+\int_{0}^{x}[y(t)]^{3} d t$.

A comparison of the approximate solution from the decomposition method with the exact solution $y(x)=e^{x}$ of the integral equation at $x=0,0.2,0.4,0.6,0.8$, and 1.0 yields the errors displayed in Table 3.2.

We can see also from Figure 3.2 that the approximation is very good. The solid curve, which represents the approximate solution almost coincides with the analytic solution (rhombuses curve).

Problem 3.3. We apply the modified decomposition method

$$
y(x)=x-x \sin x \cos x+\frac{3}{4} x^{2}+\frac{1}{4} \sin ^{2} x-\int_{0}^{x}(x-t) y(t) d t-\int_{0}^{x}(x+t)[y(t)]^{2} d t
$$

Let $y_{0}(x)=x$, then

$$
\begin{aligned}
y_{1}(x)= & -x \sin x \cos x+\frac{3}{4} x^{2}+\frac{1}{4} \sin ^{2} x-\int_{0}^{x}(x-t) y_{0}(t) d t \\
& -\int_{0}^{x}(x+t)\left[y_{0}(t)\right]^{2} d t \\
y_{2}(x)= & -\int_{0}^{x}(x-t) y_{1}(t) d t-\int_{0}^{x}(x+t)\left[2 y_{0}(t) y_{1}(t)\right] d t, \\
y_{3}(x)= & -\int_{0}^{x}(x-t) y_{2}(t) d t-\int_{0}^{x}(x+t)\left[2 y_{0}(t) y_{2}(t)+y_{1}^{2}(t)\right] d t,
\end{aligned}
$$


TABLE 3.3

\begin{tabular}{c|cccccc}
\hline$x$ & 0 & 0.2 & 0.4 & 0.6 & 0.8 & 1.0 \\
\hline Error & $2.4 \times 10^{-7}$ & $1.8 \times 10^{-5}$ & $1.8 \times 10^{-5}$ & $3.2 \times 10^{-5}$ & $6.4 \times 10^{-5}$ & $4.6 \times 10^{-3}$ \\
\hline
\end{tabular}

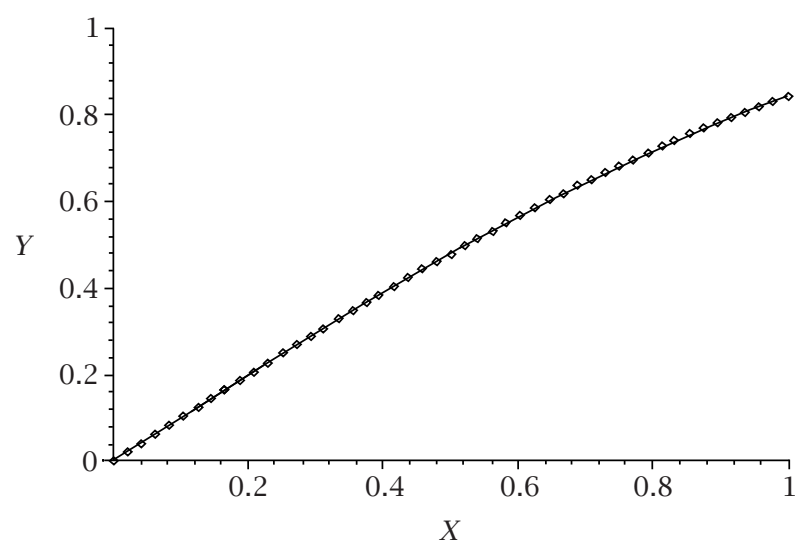

FIGURE 3.3. Decomposition method versus analytic solution for $y(x)=x-$ $x \sin x \cos x+(3 / 4) x^{2}+(1 / 4) \sin ^{2} x-\int_{0}^{x}(x-t) y(t) d t-\int_{0}^{x}(x+t)[y(t)]^{2} d t$.

$$
\begin{aligned}
y_{4}(t)= & -\int_{0}^{x}(x-t) y_{3}(t) d t-\int_{0}^{x}(x+t)\left[2 y_{0}(t) y_{3}(t)+2 y_{1}(t) y_{2}(t)\right] d t, \\
y_{5}(x)= & -\int_{0}^{x}(x-t) y_{4}(t) d t \\
& -\int_{0}^{x}(x+t)\left[2 y_{0}(t) y_{4}(t)+2 y_{1}(t) y_{3}(t)+y_{2}^{2}(t)\right] d t, \\
y_{6}(x)= & -\int_{0}^{x}(x-t) y_{5}(t) d t \\
& -\int_{0}^{x}(x+t)\left[2 y_{0}(t) y_{5}(t)+2 y_{1}(t) y_{4}(t)+2 y_{2}(t) y_{3}(t)\right] d t,
\end{aligned}
$$

$y(x)$ is approximated by using seven terms of decomposition polynomials:

$$
y(x) \approx y_{0}(t)+y_{1}(t)+y_{2}(t)+y_{3}(t)+y_{4}(t)+y_{5}(t)+y_{6}(t)
$$

A comparison of the approximate solution from the decomposition method with the exact solution $y(x)=\sin x$ of the integral equation at $x=0,0.2,0.4,0.6,0.8$, and 1.0 yields the errors displayed in Table 3.3.

We can see also from Figure 3.3 that the approximation is very good. 
In this note, we presented decomposition method as an alternate method to other approximate methods to integral equations. In the above problems, the method yields accurate computable solutions with good approximation using only few terms.

ACKNOWLEDGMENTS. The first author was partially supported by the University of Houston-Downtown (UHD) Organized Research Committee (ORC) Grant-2002. The second author was partially supported by UHD ORC Grant-2002.

\section{REFERENCES}

[1] G. Adomian, A review of the decomposition method and some recent results for nonlinear equations, Math. Comput. Modelling 13 (1990), no. 7, 17-43.

[2] _ Solving Frontier Problems of Physics: The Decomposition Method, Fundamental Theories of Physics, vol. 60, Kluwer Academic Publishers Group, Dordrecht, 1994.

[3] Y. Cherruault, G. Saccomandi, and B. Some, New results for convergence of Adomian's method applied to integral equations, Math. Comput. Modelling 16 (1992), no. 2, 8593.

[4] E. Y. Deeba and S. A. Khuri, A decomposition method for solving the nonlinear Klein-Gordon equation, J. Comput. Phys. 124 (1996), no. 2, 442-448.

[5] E. Y. Deeba, S. A. Khuri, and S. Xie, An algorithm for solving a nonlinear integro-differential equation, Appl. Math. Comput. 115 (2000), no. 2-3, 123-131.

[6] L. Gabet, The theoretical foundation of the Adomian method, Comput. Math. Appl. 27 (1994), no. 12, 41-52.

[7] A. M. Wazwaz, A reliable modification of Adomian decomposition method, Appl. Math. Comput. 102 (1999), no. 1, 77-86.

[8] S. Yalçinbaş, Taylor polynomial solutions of nonlinear Volterra-Fredholm integral equations, Appl. Math. Comput. 127 (2002), no. 2-3, 195-206.

Elias Deeba: Department of Computer and Mathematical Sciences, University of HoustonDowntown, Houston, TX 77002, USA

E-mail address: deebae@dt.uh.edu

Shishen Xie: Department of Computer and Mathematical Sciences, University of HoustonDowntown, Houston, TX 77002, USA

E-mail address: xies@dt.uh.edu 


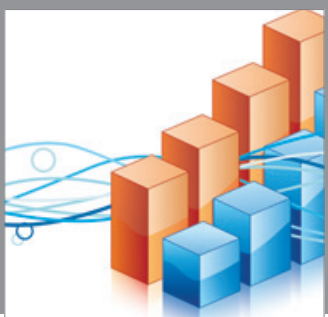

Advances in

Operations Research

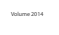

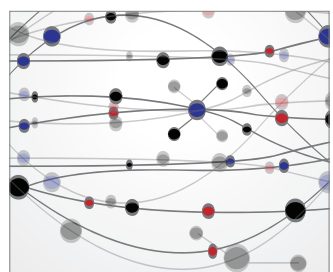

\section{The Scientific} World Journal
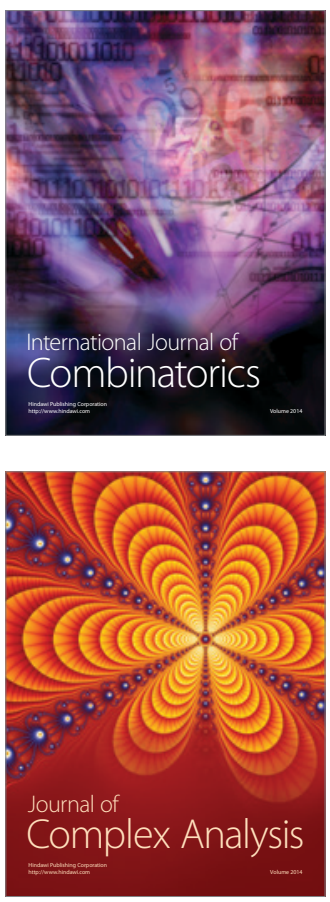

International Journal of

Mathematics and

Mathematical

Sciences
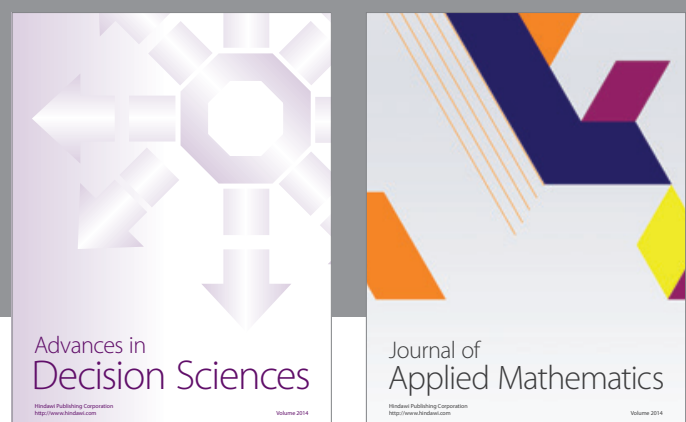

Journal of

Applied Mathematics
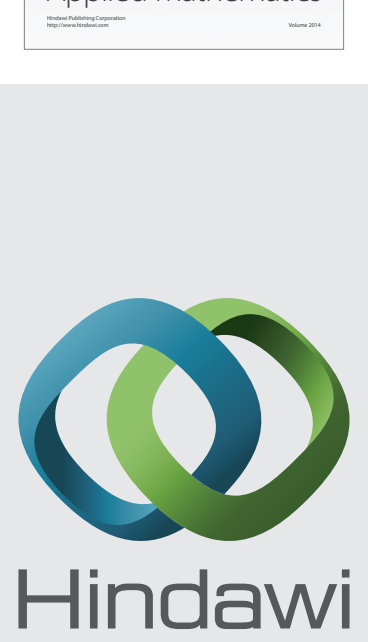

Submit your manuscripts at http://www.hindawi.com
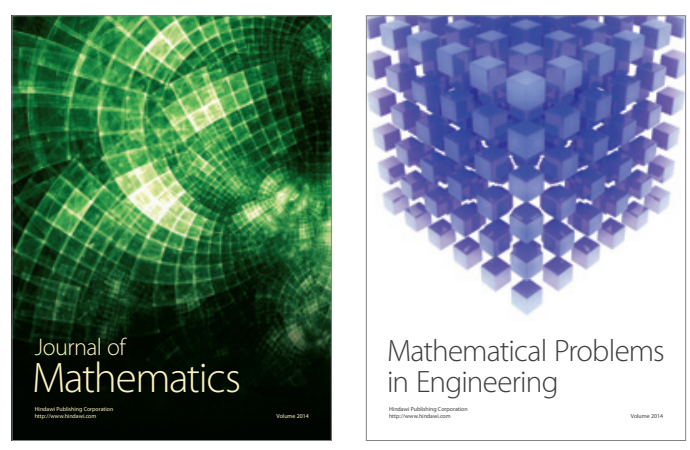

Mathematical Problems in Engineering
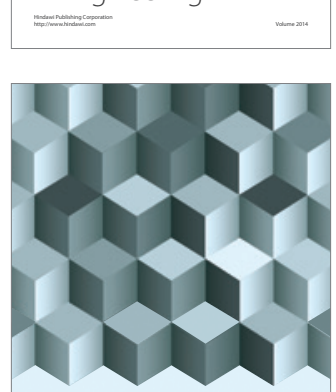

Journal of

Function Spaces
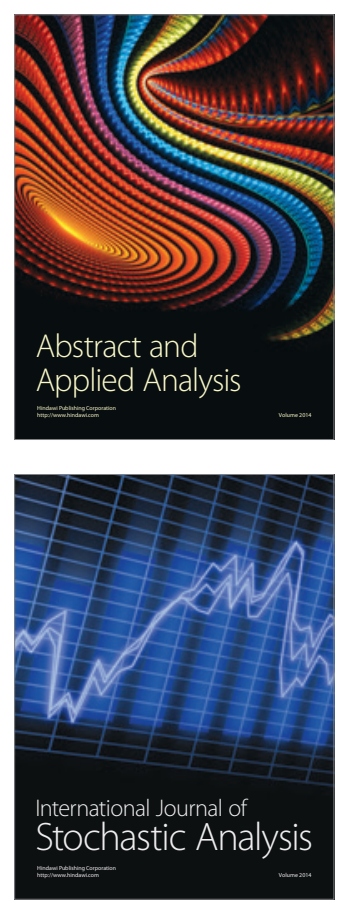

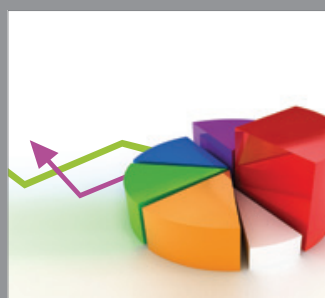

ournal of

Probability and Statistics

Promensencen
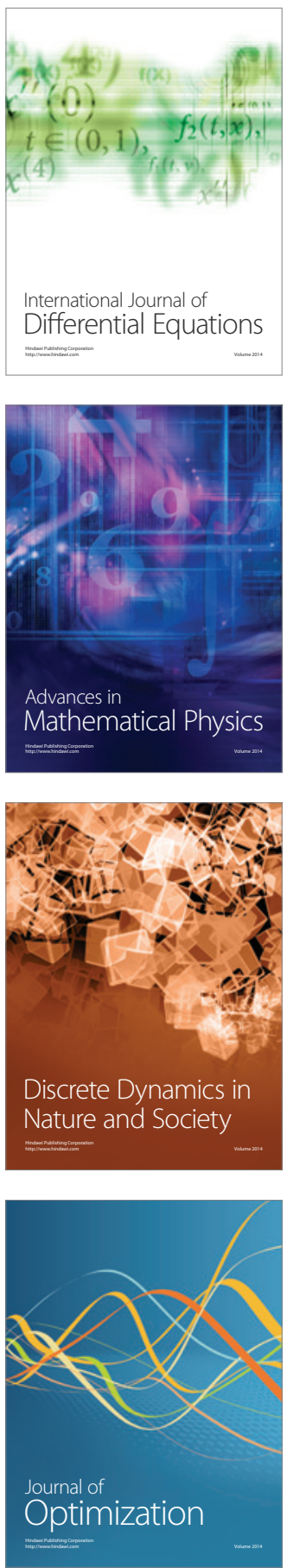